\title{
Research on the Rheological Properties of the Plant Oil Pitch
}

\author{
Weishuai Ji, ${ }^{1}$ Dongpo He ${ }^{D},{ }^{1}$ Di Wu$^{2}{ }^{2}$ and Maryam Kargar Razi $\mathbb{D}^{3}$ \\ ${ }^{1}$ School of Civil Engineering, Northeast Forestry University, Haerbin 150040, China \\ ${ }^{2}$ Longjian Road and Birdge Company Limited, Haerbin 150009, China \\ ${ }^{3}$ Faculty of Chemistry, North Branch of Tehran, Islamic Azad University, Tehran, Iran \\ Correspondence should be addressed to Dongpo He; weilg1982@163.com and Maryam Kargar Razi; m_kargarrazi@iau-tnb.ac.ir
}

Received 11 February 2021; Revised 12 March 2021; Accepted 5 April 2021; Published 17 April 2021

Academic Editor: Alireza Baghban

Copyright (c) 2021 Weishuai Ji et al. This is an open access article distributed under the Creative Commons Attribution License, which permits unrestricted use, distribution, and reproduction in any medium, provided the original work is properly cited.

\begin{abstract}
In the analysis of the rheological properties of the plant oil pitch, the original analysis method has a narrow range of analysis indicators, which affects the reliability of the analysis results. In this study, a new analytical method for the rheological properties of the plant oil pitch was designed. The microstructure characteristics of the plant oil pitch were obtained using a high-power microscope, the viscosity of the plant oil pitch was measured, and the ductility of the plant oil pitch was analyzed by setting the analysis index. The above parts are integrated, and the dynamic shear tester is used to complete the analysis of pitch rheological properties. So far, the analysis method for the rheological properties of the plant oil pitch has been designed. It can be seen from the comparison of the experimental links that this analysis method is better than the original analysis method in terms of the scope of analysis indicators. In conclusion, the analytical method of the rheological properties of the plant oil pitch is more reliable.
\end{abstract}

\section{Instruction}

The emergence of expressways is intended to serve the automobile traffic, which has the characteristics of strong transportation capacity, fast speed, and high safety, and is gradually being invested and constructed by more and more people $[1,2]$. In recent years, in order to alleviate the resource shortage of petroleum pitches, more and more attention has been paid to the development of new alternative resources such as biological pitches. Bio pitch is a kind of dark brown, non-petroleum-based bio binder, and all kinds of agricultural and forestry wastes and domestic wastes may become its raw materials. According to different sources, they are mainly divided into two categories: one is animal fat, and the other is plant-based pyrolysis products [3]. In order to master the composition and structure of biological pitches, many scholars have done a lot of research on them by means of chemical analysis and performance test. A series of mixed biological pitches and pitches were evaluated by adding biological pitches to common petroleum pitches, and the feasibility of replacing petroleum pitches was proved [4].

The plant oil pitch is a kind of waste [5] after acidification, hydrolysis, distillation, and extraction of fatty acids from vegetable oil feet. Because of its color like a pitch, it is called the plant oil pitch. However, with the rapid development of biochemical technology and the continuous progress of science and technology, the production of the plant oil pitch is gradually transformed into the large-scale production of sugar extraction from plant straw, which provides conditions for meeting the market demand of petroleum pitch. The price of the plant oil pitch is about 3000 yuan per ton, while that of the petroleum pitch is about 5000 yuan per ton. If the plant oil pitch is mixed with the plant oil pitch, the cost of the pitch mixture can be reduced, so as to reduce the cost of road construction.

The plant oil pitch has some advantages that the matrix pitch does not have, such as low carbon content and low pollution. In recent years, with the development of biochemical technology, the source of the plant oil pitch has become more and more extensive. At first, there were mainly biodiesel and animal and vegetable oils. The plant oil pitch obtained by these two methods is also called black foot, which is mainly used in the production of rubber softener, surface activated carbon, casting binder, etc. Some of the products are mainly used to make leather auxiliaries, black printing ink, and pitch coating. At present, the most popular 
production method is to use biochemical alcohol for extraction. This method can not only use the plant straw after treatment for extraction and reduce the pollution caused by straw combustion, but also extract sustainably with high yield. More importantly, the quality of this product is often higher than the products obtained by the first two production methods, so it provides the possibility for the application of the plant oil pitch in road engineering.

Different from the traditional petroleum pitch, the plant oil pitch is not a complex compound of polyaromatic rings. It has the advantages of having low carbon, environmental protection, and being renewable. In the future, after the exhaustion of petroleum pitches, it can still be extracted from plants. In addition, the plant oil pitch has a good fluidity at a lower temperature. Adding it to the petroleum pitch can not only increase the consistency of the pitch, but also increase its cohesiveness with the ore, which is more conducive to the mixing and construction of the pitch. What is more, petroleum pitches are non-degradable, and the waste pitches produced by Pavement Renovation have low utilization rate and are difficult to deal with. In the process of using, pitch smoke also contains a lot of carcinogens, which pollutes the environment and emits a lot of carbon dioxide. Therefore, people need to look for alternatives or renewable and even environmentally friendly pitches. The emergence and large-scale production of the plant oil pitches provide a new way to alleviate the industry's dependence on petroleum pitches.

The study in [6] evaluated the functional characteristics (foaming, emulsifying, and rheological properties), proteomics, and metabonomics composition of natural foaming and currently undeveloped byproducts (concentrate) in the fermentation process of vegetable oil pitch. This study highlights the potential of functional extracts extracted from the fermentation process of vegetable oil pitch as new ingredients for the preparation of sustainable food, highlights the complexity and specificity of the functional properties of the centers, and reports the contribution to mycelial structure and interfacial active molecules. The study in [7] proposed that the rheological properties of vegetable oil pitch are very important, because they affect the texture, processing performance, and stability of food.

In order to further study the plant oil pitch, the rheological properties of the plant oil pitch will be studied in this paper. The so-called rheological property refers to the quantitative relationship between strain and stress of an object under the action of external force [8]. This kind of strain (flow or deformation) is related to the nature and internal structure of the object, as well as the relative motion state between the particles in the object. For example, the rheological properties of the colloidal system are not only the reflection of the properties of individual particles, but also the result of the interaction between particles, as well as between particles and solvents. Therefore, different substances have different rheological properties. Through the study of this performance, the practical application of the plant oil pitch can be based on the theoretical basis.

\section{Materials and Methods}

The amount of the plant oil pitch has been determined in previous studies. The amount of the plant DC oil pitch is $10 \%$ of the matrix pitch, and the amount of the plant SHB oil pitch is $15 \%$ of the matrix pitch. The specific research process will not be discussed in this paper. This design mainly studies the blending mechanism and technology of the mixed plant oil pitch and determines the rheological properties of the plant oil pitch. The design circuit of performance analysis method is as follows.

Through technical streamline indicated in Figure 1, the design of the analysis method is completed. In this paper, the shear rate and heating temperature are studied. The blending temperature was determined by thermal analysis, and the shear rate was determined by observing the dispersion of the plant oil pitch in the matrix pitch at different rotating speeds by AFM. The rheological properties of the plant oil pitch were analyzed with the above technology.

\subsection{Acquisition of Microstructure Characteristics of the Plant} Oil Pitch. Due to the lack of relevant research on the rheological property analysis of the plant oil pitch, we do not know the performance of the plant oil pitch mixed with the matrix pitch. It is not clear whether the different content of pitch will affect the rheological properties of pitch. Therefore, the research on the composition of the plant oil pitch is completed first. The plant oil pitch is different from the petroleum pitch commonly used in roads, which is soluble in water and sensitive to temperature [9]. At room temperature $\left(10-20^{\circ} \mathrm{C}\right)$, the plant oil pitch is in a semi-solid gelled state, and at $40^{\circ} \mathrm{C}$, it is in a flow plastic state. When the temperature rises to about $60^{\circ} \mathrm{C}$, it is basically in a fluid state. The component extraction indexes of the plant oil pitch are shown in Table 1.

Combined with other research literature and practical engineering experience, the microstructure of the plant oil pitch extracted above was obtained by microscope and analyzed. SEM is usually used to observe the microstructure of materials, and its imaging principle is as follows.

The electron emitted by the electron gun irradiates the sample surface to be analyzed in the form of grating scanning. The image of secondary electron and backscattered electron produced by the interaction of incident electron and surface material of the sample is used to obtain the microstructure and appearance characteristics of the sample surface [10]. SEM has been widely used in various research fields, such as biology, medicine, metallurgy, chemistry, materials, and so on. This method can be used to analyze the distribution of modifier in pitch and the interface combination of modifier and pitch. In this paper, Hitachi 5-3400N type II SEM was used to scan plants and analyze their microstructure. Scanning the plant oil pitch with SEM, the results are shown in Figure 2.

Through observation, it is found that the particles in the plant oil pitch are in the form of network structure, and there are long flexible segments between the cross linking points. They make pitch particles closely together and play their 


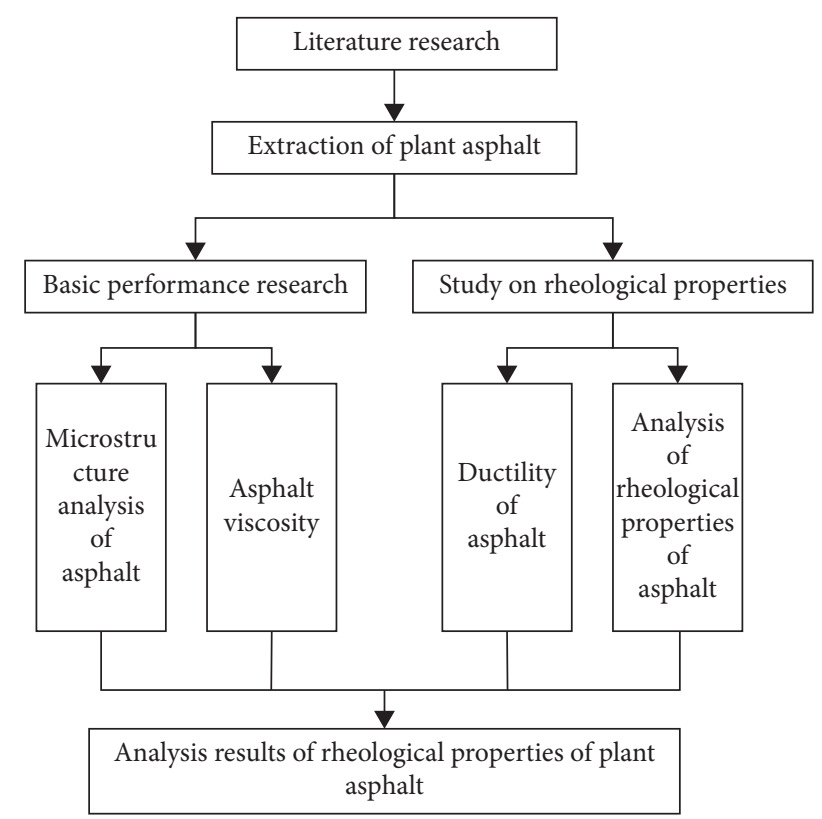

FIGURE 1: Technical route design of rheological property analysis of plant asphalt.

respective advantages together, so as to achieve the liquid state. Because of its irregular particle shape, uneven surface, and being full of large and small folds and pores, the surface area has been increased, which means that there is a large bulge area between the pitch particles, so the molecular force between the two is greatly increased, which improves the adhesion ability of pitch. At the same time, more light components in pitch (mainly oil wax) [11] changed pitch from sol structure to sol-gel structure, which reduced pitch's temperature sensitivity. It can be seen from Figure 2 that the surface of plant particles is wrapped by pitch, forming a concave convex shape on the surface, so that pitch particles are closely bonded together, so that pitch can play a good blending effect. Due to the different modulus of pitch particles, rubber particles will deform relatively large under the action of force at low temperature. Pitch particles work and consume a lot of energy, so the impact strength and plasticity of pitch particles at low temperature are improved. Using the microstructure and the viscosity of the plant oil pitch, the relevant properties of the plant oil pitch can be obtained.

2.2. Viscosity of the Plant Oil Pitch. Through the above, the microstructure of the plant oil pitch was analyzed. In order to obtain the overall performance of the plant oil pitch, the viscosity of the plant oil pitch was determined by Zhaoqing's equipment and calculation. The methods for measuring the rheological properties of fluids can be divided into capillary method, falling body method, rotation method, plate method, and vibration method. For Newtonian fluids, the Newtonian viscosity can be known by measuring the corresponding response of Newtonian fluids when a certain stress or strain is applied. For non-Newtonian fluids, the measured viscosity is called the apparent viscosity [12].
The plant oil pitch is a solid at room temperature. As the temperature increases, it gradually changes into a liquid. At this time, it can be tested for viscosity. However, at higher temperature, the viscosity measurement must be protected by inert gas to prevent the plant oil pitch from being oxidized by air. Most researches on the rheological properties of the plant oil pitch focus on the concept of apparent viscosity. The change of the apparent viscosity of the plant oil pitch with temperature at a fixed shear rate is as follows, and its change with shear rate at a constant temperature is also listed.

The specific method of measuring viscosity with capillary viscometer is as follows: the liquid is forced through the thin cavity tube. The viscosity of the liquid is determined according to the measured volume flow rate, applied pressure, and pipe size. Common capillary viscometers can be divided into three main types: glass capillary viscometer, cylinder piston (or plug rod) combined viscometer, and sharp hole viscometer [13]. The first is mainly used to directly measure the kinematic viscosity of low viscosity Newtonian fluid; the second is mainly used to measure the viscosity of thicker non-Newtonian fluid; the third is a kind of industrial viscometer. Capillary viscometer is a general method to study the rheological behavior of melt with large molecular weight. This method has many advantages; especially its measuring conditions are close to the processing conditions of extrusion and injection. In addition to the measurement of viscosity and flow characteristics, the elasticity of melt can be estimated from the data of extrusion swell, and the unsteady flow phenomenon can be studied. The main disadvantage is that the shear velocity changes unevenly along the radial direction of the capillary. In order to get the correct viscosity value, some correction must be made. When measuring low viscosity samples at low shear rate, the self-weight outflow makes the sheer force low. The plant oil pitch was determined by glass capillary viscometer. The equipment selected in this study is shown in Figure 3.

The rheological properties of the plant oil pitch were analyzed and characterized by a thermal analyzer [14], and the relationship between its softening point and rheological parameters was obtained. The plant oil pitch was analyzed and characterized by the tube pressure thermodynamics analyzer. The experimental method is fast and repeatable. In the use of the instrument, the glass transition temperature, softening point, and rheological properties of the plant oil pitch can be measured simultaneously. The device is used to measure the ordinary plant oil pitch in Australia. The results show that the plant oil pitch behaves as Bingham fluid when the pressure is $100 \mathrm{~Pa}$ and the temperature is higher than the softening point. Therefore, the high temperature and high pressure viscometer is modified and designed, which has good consistency with the conventional viscometer.

The viscosity characteristics of the corresponding plant oil pitch are obtained by the viscometer. The plant oil pitch is composed of a large number of aromatic molecules with different degrees of polymerization. The molecular weight and structure are different, and the properties of pitch are the comprehensive reflection of these different components, which makes the characterization of pitch properties very 
TABLE 1: Index of plant asphalt extract.

\begin{tabular}{lccc}
\hline Testing items & Test method & Reference requirement & Detection result \\
\hline Dynamic viscosity (Pa.s) & T0620 & $\geq 360$ & 550 \\
Flash point $\left({ }^{\circ} \mathrm{C}\right)$ & T0611 & $\geq 240$ & $>240$ \\
Volatility $(\%)$ & - & $\leq 1$ & $\leq 1$ \\
Density $(\mathrm{g} / \mathrm{cm})$ & T0603 & $1.300-1.500$ & 1.254 \\
\hline
\end{tabular}

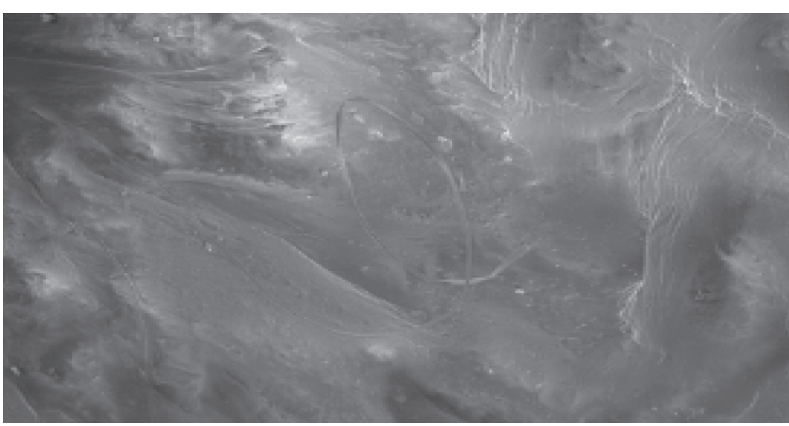

(a)

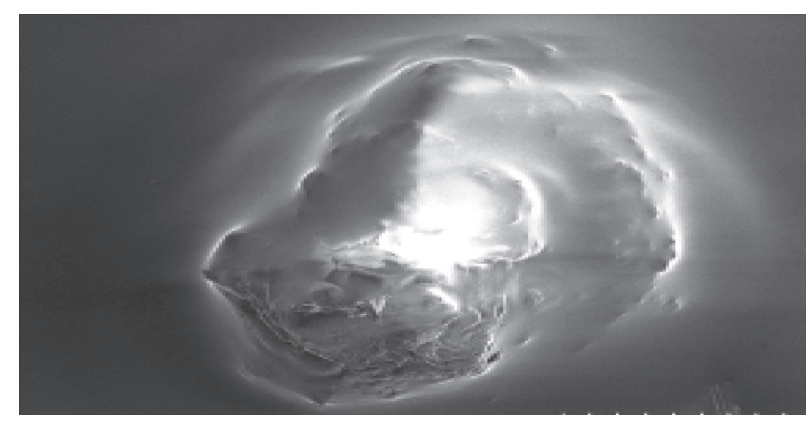

(b)

FiguRE 2: Electron micrograph of plant asphalt. (a) Low power microscopic results. (b) High power microscopic results.

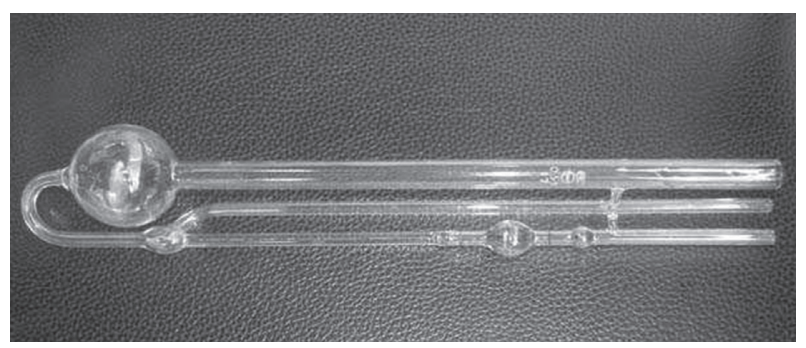

Figure 3: Capillary viscometer.

difficult. Generally, there are two ways to characterize its rheological properties: one is to measure the viscosity of the plant oil pitch with the change of temperature at a fixed shear rate; the other is to measure the viscosity with the change of shear rate at a constant temperature. The fluidity of the plant oil pitch is controlled by the specific flow range and effective viscosity. When the heating temperature and mechanical force are increased, the plant oil pitch will gradually become Newtonian fluid, and its rheological properties only depend on the viscosity of pitch system. In this temperature range, the rheological properties of the plant oil pitch can be easily characterized and controlled. The relationship between viscosity of pitch and temperature can be calculated by the following formula:

$$
\eta=Q r^{Y p_{i}}
$$

where $\eta$ is the viscosity of the plant oil pitch, $Q$ is the regression constant, $Y$ is the gas constant, and $p_{i}$ is the viscous flow activation energy. $p_{i}$ means that a molecule overcomes the energy required by the surrounding molecules to move the position, and its value reflects the temperature dependence of the melt viscosity. The larger the $E$ is, the more sensitive the viscosity of the asphalt is to temperature changes; at the same time, the lager the $p_{i}$ value is, the greater the melt viscosity is.

There is an exponential relationship between pitch viscosity and temperature, which is due to the change of activation energy of viscous flow. When the heating temperature is increased, pitch continuously changes into a new form. During the transition from the original glass state to the flowing liquid state, the structure of pitch obviously changes, which is related to the weakening of the intermolecular bond and the fracture of the bridge bond of the outer molecular structure. In pitch, the addition of additives (such as furfural, kerosene, toluene, oleic acid, and warin) can greatly reduce the viscosity. Therefore, adding surfactants or solvents to improve the rheological properties of the plant oil pitch has attracted extensive attention.

In addition to the above equipment and calculation process, in order to ensure the accuracy of the plant oil pitch viscosity, it is necessary to add some equipment to ensure the validity of the measurement results. The specific equipment is as follows.

Heating system: when the viscosity value of the plant oil pitch is high, it is necessary to measure its viscosity within the temperature range of $100-250^{\circ} \mathrm{C}$. NDJ-79 viscometer and NDJ-99 viscometer are only equipped with water bath heating system. In order to determine the high temperature viscosity of the plant oil pitch, we need to design and install the high temperature heating system ourselves. For this reason, the resistance furnace heating device is specially designed for the two kinds of viscometers. The heat conducting medium is glycerin. It is found that there are some problems in the heating system, such as inaccurate temperature control and uneven temperature of the plant oil pitch system. In view of the above problems, NDJ-31 viscometer is specially equipped with HSG-1 high temperature constant temperature bath, which can be used to measure 
the viscosity of the plant oil pitch material with the temperature below $280^{\circ} \mathrm{C}$, and the heat conducting medium is high temperature heat conducting silicone oil.

Display of control system and viscosity measurement value: NDJ-79 viscometer [15] is mechanically controlled, and its viscosity value is read directly from the dial. In the whole measurement process, the computer is used to control the NDJ-99 viscometer, and its viscosity value is directly displayed by numerical value. The viscosity of NDJ-79 viscometer is mainly displayed by pointer meter, while that of NDJ-99 viscometer is mainly displayed by digital meter. NDJ-31 viscometer adopts microcomputer to control and process data, and its viscosity value is directly displayed by numerical value and can be printed [16].

Rotor shape: the shape of the rotor used in the viscometer is shown in Figure 4. The rotor of NDJ-79 viscometer is hollow cylinder, the rotor of NDJss-99 viscometer is solid cylinder, and the rotor of NDJ-31 viscometer is solid cone.

The rheological behavior of the plant oil pitch changes rapidly with the increase of the molecular weight of the plant oil pitch (increased degree of thermal polymerization). After the plant oil pitch is fully melted and liquefied, the plant oil pitch system shows Newtonian rheological characteristics, but with the heat treatment, it shows viscoelasticity [17]. Although the rheological properties of different pitches are different, the general trend is similar. That is to say, the plant oil pitch is melted into a liquid state and then gradually solidified into coke after the intermediate phase. The viscosity of the plant oil pitch was determined by the above parts.

2.3. Ductility of the Plant Oil Pitch. In the above, the viscosity of the plant oil pitch was measured, and the basic performance analysis of the plant oil pitch was completed. There are 6 common types of the plant oil pitch, and the specific performance analysis indicators are shown in Table 2.

Through the above analysis, the corresponding analysis results are obtained. Comparing the above indexes, it can be seen that the penetration curve of pitch sample changes with the penetration time under different temperature and load. The curves are inversely extended and intersected at $(0,0)$ point, as shown in Figure 5.

The obtained curve shape is actually the creep time curve of pitch. The initial stage is a "migration stage" of rapid deformation and growth, followed by a "stable stage" of gradually approaching linear growth. But it is different from creep test, because creep test has fixed load and load area no matter compression, bending, splitting, and shear, so it has the same stress and basically constant strain rate. Although the penetration test is similar to shear creep, but the load area is needle like and gradually penetrated into the pitch, so although the load is fixed, the stress and strain rate decrease with the increase of penetration depth. Therefore, penetration test is not a simple creep test, but a real description of pitch rheological properties.
By analyzing the test data, it is found that when the temperature and load conditions are constant, the process of penetration with penetration time conforms to the following relationship:

$$
\lg P=B * \lg t+j,
$$

where $P$ is the penetration $(0.1 \mathrm{~mm}), t$ is the penetration time $(\mathrm{s})$, and $j$ is the measurement constant.

According to the above curve image, the $B$ value is the slope of the rheological curve, which reflects the speed of growth with the increase of penetration time, which can be called the penetration time index. The larger the $B$ value is, the larger the slope of the rheological curve is, and the larger the shear deformation is in the same load time; on the contrary, the smaller the Bvalue is, the smaller the deformation is in the same time. It reflects the time sensitivity of the penetration, and its significance is similar to the $b$ value (compound fluidity or rheological index) when the rheometer is used to study the rheological properties. The ductility index of the plant oil pitch can be obtained by using the above formula.

\subsection{Dynamic Shear Tester Is Used to Analyze the Rheological} Properties of Pitch. Based on the structure, viscosity, and ductility of the plant oil pitch obtained in the above part, dynamic shear tester was used to complete the rheological property analysis of pitch. The viscosity of pitch under dynamic load is less than that under static load. Therefore, in this study, the measurement and analysis of the rheological properties of the plant oil pitch has become an important topic. At present, dynamic shear rheological test is widely used at home and abroad. Through the dynamic shear test of pitch binder, the rheological properties of pitch were evaluated by the test results.

Dynamic shear rheometer (DSR) is a basic test instrument for viscoelasticity of polymer materials [18]. The viscoelasticity of pitch is described by the complex shear modulus $G^{l}$ and phase angle $\wp$ of the binder measured by DSR. The complex shear modulus $G^{l}$ can be used to measure the total resistance of the material under repeated shear, which can be expressed as the ratio of the maximum shear stress to the maximum shear strain, as shown in the following formula. It is the complex sum of real number $G^{i}$ and imaginary number $G^{i i}$, as shown in formula (3). Among them, the real part $G^{i}$ is the dynamic elastic modulus, that is, the elastic (recoverable) part, which reflects the energy stored in the process of pitch deformation, so it is also called the storage elastic modulus. The imaginary part $G^{i i}$ is called the lost elastic modulus, that is, the viscous (nonrecoverable) part, which reflects the energy lost by the work of pitch in the process of shear deformation. The phase angle $\wp$ is the ratio of elastic and viscous components of pitch binder, as shown in formula (4). Due to the influence of viscous components, the above index relations can be expressed in complex coordinates. The relationship between, $G^{l} G^{i}, G^{i i}$, and $\wp$ can be expressed as 


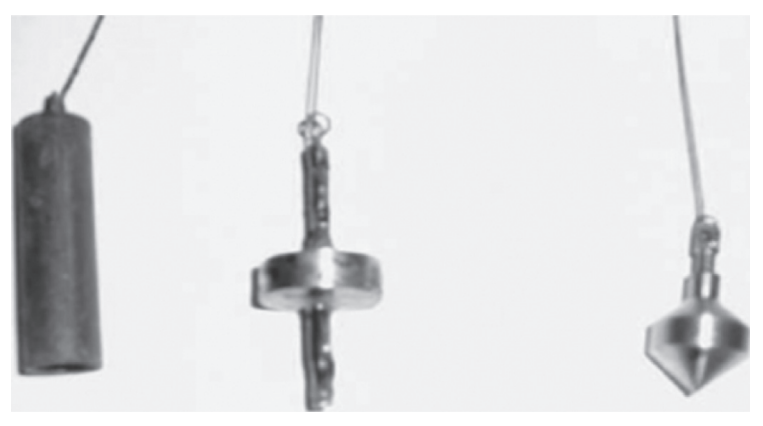

(a)
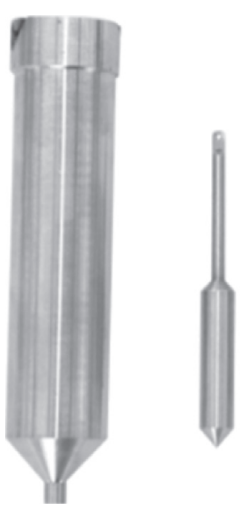
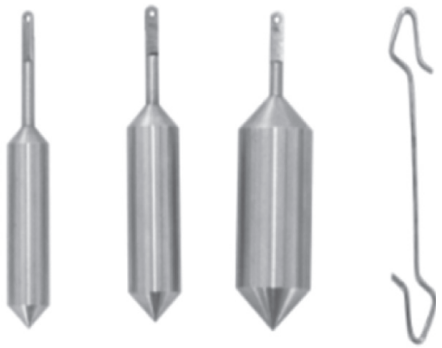

(b)

Figure 4: Shape of viscometer rotor. (a) Traditional rotor shape. (b) The rotor shape is selected in this paper.

TABLe 2: Performance analysis index of plant asphalt.

\begin{tabular}{|c|c|c|c|c|c|c|}
\hline $\begin{array}{l}\text { Asphalt } \\
\text { numbering }\end{array}$ & $\begin{array}{c}\text { Softening point } \\
\left({ }^{\circ} \mathrm{C}\right)\end{array}$ & $\begin{array}{l}\text { Toluene insolubles } \\
\text { (\%) }\end{array}$ & $\begin{array}{l}\text { Quinoline insoluble } \\
(\%)\end{array}$ & Resin (\%) & $\begin{array}{c}\text { Coking value } \\
(\%)\end{array}$ & Ash content (\%) \\
\hline Plant pitch 1 & 109.5 & 30.68 & 10.73 & 2.51 & 59.1 & 0.15 \\
\hline Plant pitch2 & 106.2 & 30.30 & 9.30 & - & - & - \\
\hline Plant pitch 3 & 105.2 & 31.20 & 11.70 & - & - & - \\
\hline Plant pitch 4 & 104.5 & 34.68 & 12.73 & 3.20 & - & 0.21 \\
\hline Plant pitch 5 & 108.5 & 30.60 & 11.40 & - & - & 0.18 \\
\hline Plant pitch 6 & 103.4 & 32.20 & 11.70 & 1.2 & - & 0.17 \\
\hline
\end{tabular}

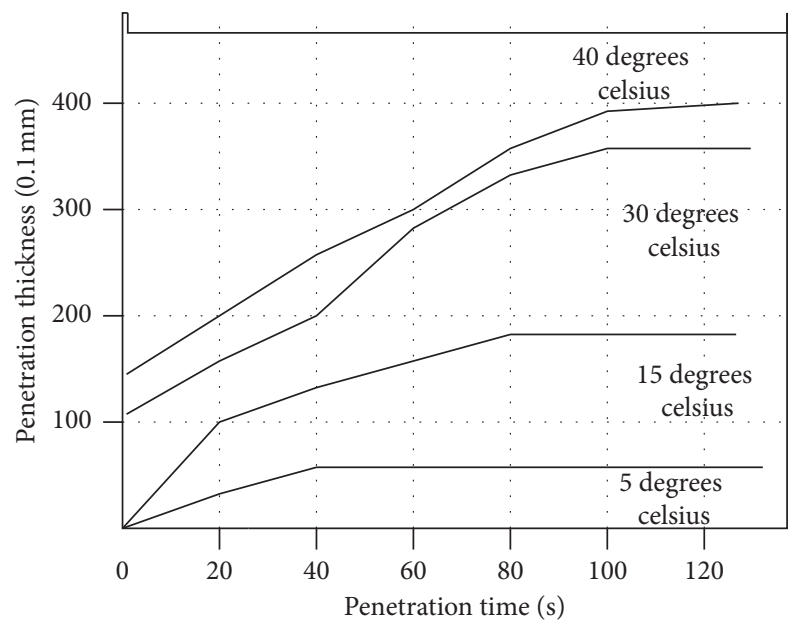

FIGURE 5: Ductility curve of plant asphalt.

$$
\begin{aligned}
G^{l} & =\frac{\Im \max }{\Re \max }, \\
G^{l} & =G^{i}+G^{i i}, \\
\tan \wp & =\frac{G^{i i}}{G^{i}}, \\
G^{i} & =G^{l} \cos \wp,
\end{aligned}
$$

$$
G^{i i}=G^{l} \sin \wp \text {. }
$$

The dynamic shear rheometer used in this study belongs to the plate rheometer, and the specific equipment is shown in Figure 6. The pitch sample is between two plates with a thickness of about 1.1-2.2 mm. The bottom plate is fixed and one plate rotates around the central axis. According to the requirements of flow performance analysis, a certain shear force is applied to the sample through the continuous rotation of the upper indenter, and the test results are recorded. In the real world, there are two kinds of commonly used shear rheometer, used in pitch binder test, i.e., double cylinder rotary rheometer and flat plate rheometer. Their test principle is the same. The method of applying deformation is called control strain method, and the method of applying stress is called control stress method.

Physical MCR101 dynamic shear rheometer [19] was used to conduct temperature sweep and frequency sweep on pitch samples. Among them, the temperature range of temperature scanning test is $20^{\circ} \mathrm{C}-80^{\circ} \mathrm{C}$, and the load frequency is $10 \mathrm{rad} / \mathrm{s}$, equivalent to $1.592 \mathrm{~Hz}$. The temperature of frequency scanning is also $20^{\circ} \mathrm{C}-80^{\circ} \mathrm{C}$, and the frequency range is $0.1-100 \mathrm{rad} / \mathrm{s}$ at any temperature [20]. The chassis with a diameter of $20 \mathrm{~mm}$ is used, and the thickness of pitch sample is $10 \mathrm{~mm}$. The selected target value of control stress is $120 \mathrm{~Pa}$ for test. The first 10 cycles do not record data, and the second cycle records data, which is used to calculate the composite shear modulus and phase angle. The data acquisition system is used to record and calculate the flow performance of the plant oil pitch. 


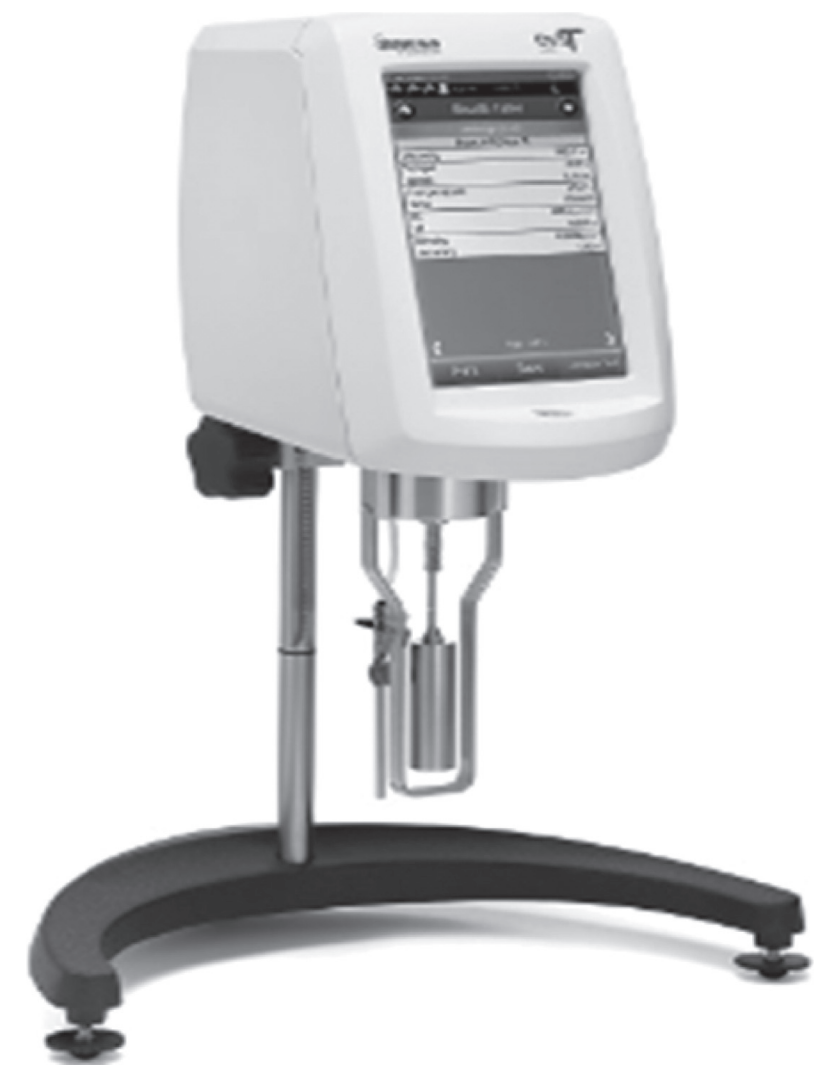

Figure 6: Dynamic rheological tester.

After the instrument analysis, the corresponding values are obtained, and the flow performance calculation and analysis process is completed according to the following formula. The pitch can flow under the action of force; when the fluid is sheared, the shear stress $F(\mathrm{~N} / \mathrm{M})$ of pitch may have the following relationship with the shear velocities $d v$ and $d w(1 / \mathrm{s})$ :

$$
F=\frac{\eta d v}{d w}
$$

where $\eta$ is the asphalt viscosity. If the viscosity of a fluid does not change with the change of shear rate, that is to say, it is a simple linear relationship, it is called "Newtonian fluid." On the contrary, the fluid whose corresponding relationship between shear rate and shear stress does not follow the corresponding relationship of the above formula is called "non-Newtonian fluid." Generally speaking, the viscosity of gas increases with the increase of temperature, and the viscosity of liquid decreases with the increase of temperature.

From the above formula, the legal unit of measurement for viscosity $\eta$ is $N \cdot s / m^{2} 111$, that is, $\mathrm{Pa} \cdot s$. At present, the units of viscosity data found in the manual are mostly poise $(P)$, which is an illegal unit. The conversion is as follows:

$$
l c p=\left\{\begin{array}{l}
10^{-2} p \frac{N \cdot s}{m^{2}} \\
10^{-3} \mathrm{~Pa} \cdot s \\
1 \mathrm{mPa} \cdot s .
\end{array}\right.
$$

For non-Newtonian fluids, the formula is still used to calculate their viscosity, which is called "apparent viscosity." The relationship between shear stress and velocity gradient (also known as shear rate) is different, so non-Newtonian fluid can be divided into pseudoplastic fluid, bulging plastic fluid, and Bingham plastic fluid. For many non-Newtonian fluids, in a large range of shear velocity, the following equation can be used to describe them. The specific formula is as follows:

$$
F=P\left(\frac{d v}{d w}\right)^{n}
$$

where $n$ is the flow characteristic index and $P$ is the consistency coefficient. Newtonian fluid is a special case of $n=1$, $p=1$. It should be noted that, for non-Newtonian fluids, $p$ is not a viscosity. Through the above formula, the test result of the tester is calculated to obtain the corresponding rheological characteristic value. So far, the analysis method for the rheological properties of the plant oil pitch has been designed.

\section{Results}

3.1. Environment. In order to verify that the analysis method designed in this paper is more effective than the original analysis method, the structure of the experimental link is used to obtain the difference between the design analysis method and the original analysis method [21]. In this experiment, it is embodied in the form of comparative analysis indicators. In order to ensure that the equipment and reagents do not affect the experimental results during the experiment, the experimental environment is set based on Figure 7.

The original method and the designed analysis method are used to analyze the experimental samples and complete the experimental process.

3.2. Sample Preparation of the Plant Oil Pitch. In the preparation of the plant oil pitch, the no. 90 matrix pitch and the plant oil pitch were heated to $135^{\circ} \mathrm{C}$. The plant oil pitches of different proportions were poured into the no. 90 matrix pitches and stirred well for about 5 minutes. The plant oil pitch and the matrix pitch were fully mixed and prepared for standby. The high-speed shear mixer is shown in Figure 8.

Under certain stirring conditions, a certain proportion of the plant oil pitch was added to the matrix pitch to form the stable blending system. In order to ensure the blending quality of the plant oil pitch, the heating temperature, shear rate, and blending time should be controlled. However, due to the friction between the plant oil pitch and the stirring blade at high shear rate, a lot of heat is generated, which makes the heating temperature difficult to control. The premise of selecting the heating temperature of blending is to ensure the good fluidity of pitch, and the plant oil pitch and matrix pitch will not age. Therefore, the heating temperature of the blend was determined to be $135^{\circ} \mathrm{C}$. The preparation of the plant oil pitch is completed with the above settings, and the specific experimental samples are shown in Figure 9. 


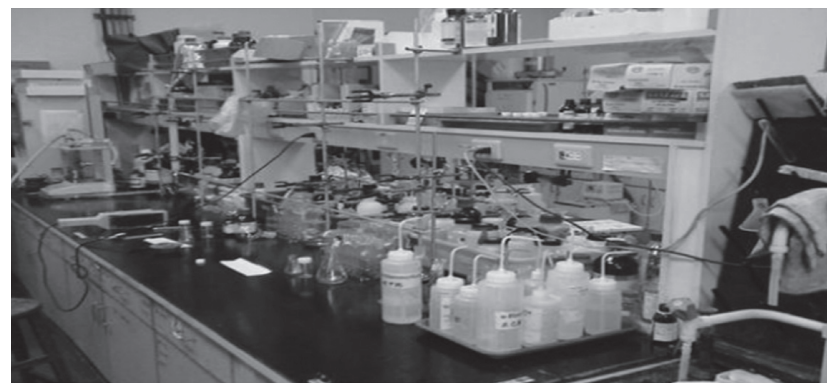

Figure 7: Experimental environment.

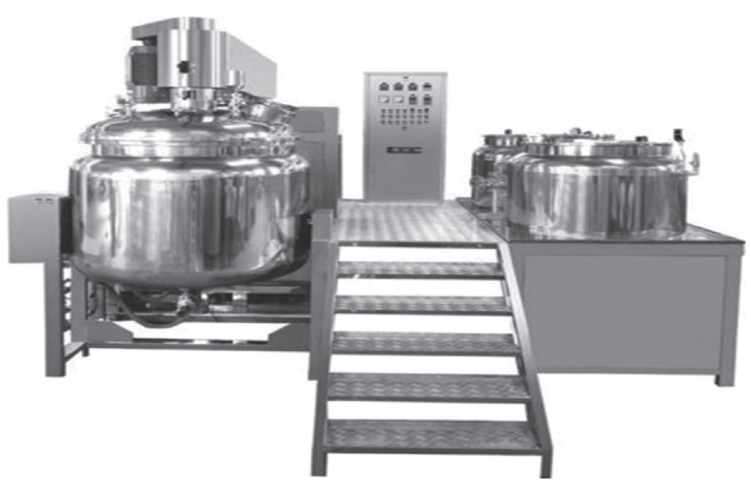

FIgURE 8: Shear mixer.

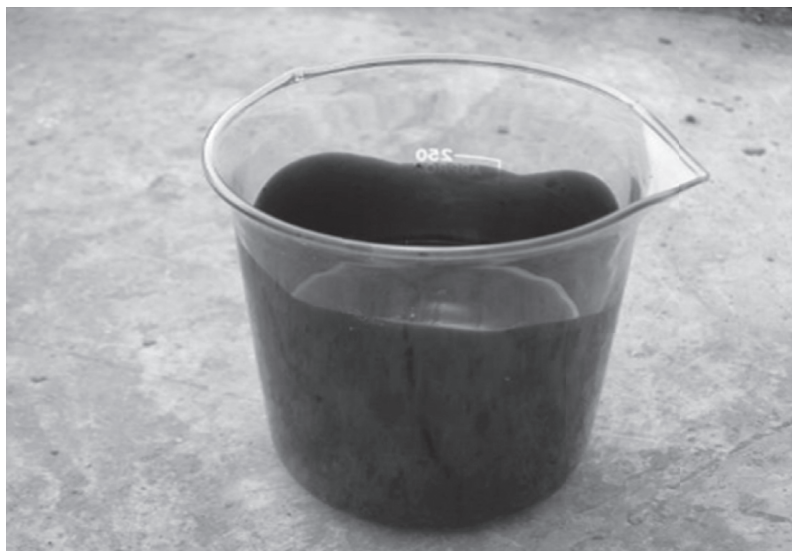

FIgURE 9: Plant asphalt sample.

The method designed in this paper and the original method are used to analyze the asphalt samples, and the analysis index ranges of the two methods are compared.

3.3. Experimental Results. Using the above experiments, the comparison between the original analysis method and the design analysis method in the article is completed. The specific comparison results are shown in Figure 10.

In order to better reflect the experimental results, graphics are used to describe the analysis indicators. In the two analysis methods, the proportion of each index is represented by a square. The shadow part is the included index, and the blank part is the missing index. From the above images, it can be seen that the original analysis method has a small selection range for the analysis index part. The index selection range of the analysis method designed in this paper is the same as that of the experiment. Therefore, the designed method has a wide range of analysis indexes, which can ensure the accuracy of the analysis results. In conclusion, the analytical method of the rational properties of the plant oil pitch designed in this paper is superior to the original analytical method.

In order to verify the analysis accuracy of the reasonable performance analysis method of vegetable oil asphalt designed in this paper, comparative experiments are carried out, and the comparison results are shown in Table 3. 


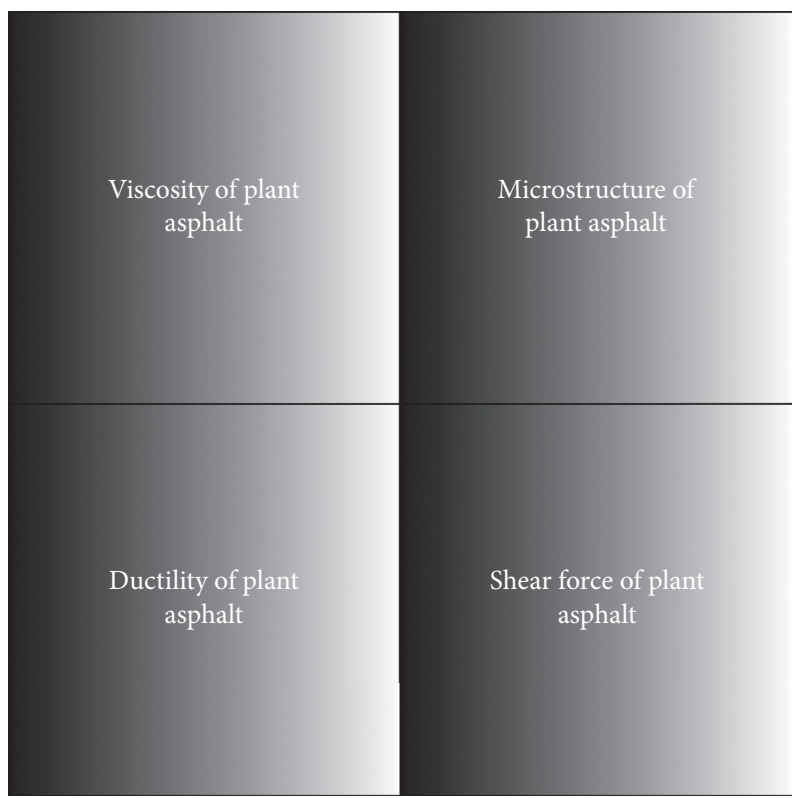

(a)

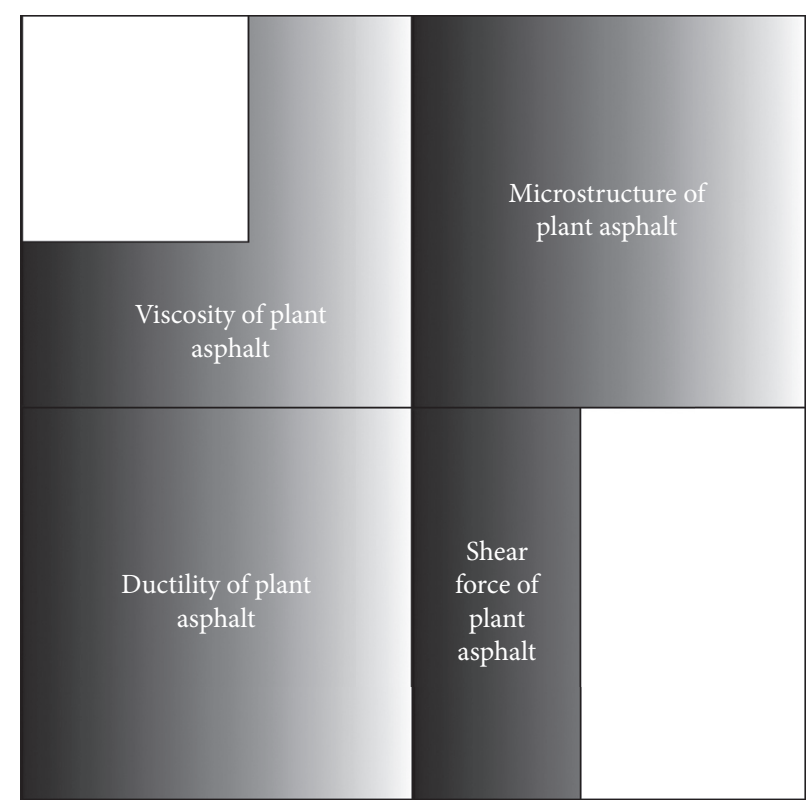

(b)

Figure 10: Comparison results between design method analysis index and original method analysis index. (a) Analysis index of design method in this paper. (b) Original method analysis index.

TABLE 3: Comparison of analysis accuracy of different methods.

\begin{tabular}{lcc}
\hline Asphalt numbering & Analysis method of this paper (\%) & The original analysis method (\%) \\
\hline Plant pitch 1 & 99.5 & 60.6 \\
Plant pitch 2 & 96.2 & 60.3 \\
Plant pitch 3 & 95.2 & 61.2 \\
Plant pitch 4 & 94.5 & 64.6 \\
Plant pitch 5 & 98.5 & 60.6 \\
Plant pitch 6 & 93.4 & 62.2 \\
\hline
\end{tabular}

It can be seen from Table 3 that, compared with the original analysis method, the analysis accuracy of the reasonable performance analysis method of vegetable oil asphalt designed in this paper is higher, which can ensure the accuracy of the analysis results.

\section{Conclusions}

The analysis index range of the existing analysis method is narrow, which affects the reliability of the analysis results. A new method for the rheological properties of vegetable oil asphalt was designed:

(1) Due to the limitation of time, manpower, and material force, this paper only discusses the rheological properties of asphalt, especially the rheological index obtained by traditional asphalt rheological test, and cannot establish the quantitative relationship between them completely. In order to make the research of this subject more perfect, we need further research in the future.

(2) The index selection range of the analysis method designed in this paper is the same as that of the experimental results. Therefore, the analysis method designed has a wide range of analysis indexes, which can ensure the accuracy of the analysis results.

(3) At present, the selection range of conventional analytical instruments is relatively narrow. In order to analyze the rheological properties of vegetable oil asphalt at higher or lower temperatures, the structure and test accuracy of permeability meter must be improved.

\section{Data Availability}

Data are available on request to the corresponding author.

\section{Conflicts of Interest}

The authors declare that there are no conflicts of interest regarding the publication of this article.

\section{References}

[1] N. S. Muhazeli, N. A. Nordin, S. A. Mazlan et al., "Characterization of morphological and rheological properties of rigid 
magnetorheological foams via in situ fabrication method," Journal of Materials Science, vol. 54, no. 21, pp. 13821-13833, 2019.

[2] T. Mohammad and N. Saeed, "Mechanical, rheological and computational study of PVP/PANI with additives," Iranian Journal of Chemical Engineering, vol. 39, pp. 281-296, 2020.

[3] I. Y. Skvortsov, L. A. Varfolomeeva, and V. G. Kulichikhin, "The effect of tetraethoxysilane on the phase state, rheological properties, and coagulation features of polyacrylonitrile solutions," Colloid Journal, vol. 81, no. 2, pp. 165-175, 2019.

[4] R. Hamid, M. Mehrdad, and G. Hormoz, "Characterization of microbubble-based drilling fluids: investigating the role of surfactants and polymers," Iranian Journal of Chemical Engineering, vol. 39, pp. 123-131, 2020.

[5] S. Shabnam, J. Aboilfazl, and R. Mehdi, "Antibacterial activity of mesoporous silica nanofibers," Iranian Journal of Chemical Engineering, vol. 39, pp. 1-11, 2020.

[6] J. Lonchamp, P. S. Clegg, and S. R. Euston, "Foaming, emulsifying and rheological properties of extracts from a Coproduct of the quorn fermentation process," European Food Research and Technology, vol. 245, no. 9, pp. 1825-1839, 2019.

[7] J. Tan, S. Martini, Y. Wang et al., "Interlaboratory measurement of rheological properties of tomato salad dressing," Journal of Food Science, vol. 84, no. 11, pp. 3204-3212, 2019.

[8] O. Seungtae, K. Seokkan, and R. Seunggeol, "Performance analysis of gravity-driven oil-water separation using membranes with special wettability," Langmuir: The ACS J. Surface. Colloid.vol. 35, pp. 7769-7782, 2019.

[9] T. Elton, C. Gustavo, and A. Ermeson, "A hierarchical approach for availability and performance analysis of private cloud storage services," Computer, vol. 100, pp. 621-644, 2018.

[10] G. Xia, F. Fang, M. Zhang, Q. Wang, and J. Wang, "Performance analysis of parametrically and directly excited nonlinear piezoelectric energy harvester," Archive of Applied Mechanics, vol. 89, no. 10, pp. 2147-2166, 2019.

[11] H. Sharma and K. S. Sandha, "Thermally aware modeling and performance analysis of MLGNR as on-chip VLSI interconnect material," Journal of Electronic Materials, vol. 48, no. 8, pp. 4902-4912, 2019.

[12] S. Mehtab, "Performance analysis of WDM-FSO system under adverse weather conditions," Photonic Network Communications, vol. 36, pp. 1-10, 2018.

[13] J. Reid, A. Bertram, and D. Topping, "The viscosity of atmospherically relevant organic particles," Nature Communications, vol. 9, p. 956, 2018.

[14] P. S. Stephanou and M. Kröger, "From intermediate anisotropic to isotropic friction at large strain rates to account for viscosity thickening in polymer solutions," The Journal of Chemical Physics, vol. 148, no. 18, Article ID 184903, 2018.

[15] D. Sandip and B. Ritabrata, "Violation of universal lower bound for the shear viscosity to entropy density ratio in dark energy dominated accretion," The European Physical Journal C, vol. 79, pp. 1-9, 2019.

[16] R. C. Hamid, R. O. Mohammad, G. Barat, and A. Mehdi, "Optimizing different angles of venturi in biodiesel production using CFD analysis," Iranian Journal of Chemical Engineering, vol. 38, pp. 285-295, 2019.

[17] O. Walter and W. Norbert, "Controlling the elongational flow behavior of complex shear-thinning fluids without affecting shear viscosity," Rheologica Acta Journal Impact, vol. 58, pp. 687-698, 2019.

[18] A. L. Proskovec, A. I. Wiesman, E. Heinrichs-Graham, and T. W Wilson, "Load effects on spatial working memory performance are linked to distributed alpha and beta oscillations," Human Brain Mapping, vol. 40, no. 12, pp. 3682-3689, 2019.

[19] H.-M. Wu, H.-Q. Jie, H. Wang et al., "A novel POU domain class 3 transcription factor 4 mutation causes X-linked nonsyndromic hearing loss in a Chinese family," Chinese Medical Journal, vol. 132, no. 18, pp. 2251-2253, 2019.

[20] A. Khalida, S. Arif, Z. Naila, and J. Kanwal, "Chemical dynamics of monodispersed iron oxide nanoparticles," Iranian Journal of Chemical Engineering, vol. 38, pp. 21-30, 2019.

[21] J. Ayari, I. J. Karoui, and M. Abderrabba, "A comparative study between different Tunisian propolis essential oils and their antioxidant activities," Iranian Journal of Chemical Engineering, vol. 39, pp. 217-231, 2020. 